\title{
PREVALENCIA DE RIESGO DE TRASTORNOS ALIMENTARIOS EN ADOLESCENTES ESCOLARIZADOS DEL NORTE DE CHILE
}

\section{PREVALENCE OF RISK OF EATING DISORDERS IN SCHOOL ADOLESCENTS FROM NORTHERN CHILE}

\author{
Alfonso Urzúa M., Sandy Castro R., America Lillo O., Carolina Leal P. \\ Escuela de Psicología \\ Universidad Católica del Norte. Antofagasta, Chile.
}

\begin{abstract}
Objective: To describe the prevalence of risk for eating disorders in adolescents from schools aged 13 to 18 years. Methods: We applied the EDI-2 to 1.429 participants from Antofagasta attending public, subsidized and private schools. Results: there are significant differences in the risk factors evaluated between men and women, age groups and kind of schools In general, $7.4 \%$ of those evaluated were at risk category, increasing to $8.9 \%$ in women. Conclusions: The prevalence of risk of eating disorders is higher among women, aged 15 to 18 years and in participants from public establishments.
\end{abstract}

Key words: eating disorders, adolescents, risk prevalence, EDI - 2.

Este trabajo fue recibido el 3 de Septiembre de 2010 y aceptado para ser publicado el 15 de Mayo de 2011.

\section{INTRODUCCIÓN}

Los Trastornos de la Conducta Alimentaria (TCA) se configuran como la tercera enfermedad crónica más común entre los adolescentes después de la obesidad y el asma (1). Éstos comprenden dos tipos de alteraciones conductuales: unos directamente relacionados con la comida y el peso y otros derivados de la relación consigo mismo y con los demás. Se caracterizan por una búsqueda deliberada de pérdida de peso, inducida y mantenida por la propia persona mediante actitudes restrictivas y/o purgativas en la alimentación o la realización excesiva de ejercicio. Junto a la Anorexia Nerviosa (AN) y la Bulimia Nerviosa (BN), se incluyen, dentro de los TCA, la categoría de TCA No Especificados para los que no cumplen los criterios propios de un trastorno específico (2).

Lo más característico de la clínica de la AN es la instauración paulatina del deseo de perder cada vez más peso y un miedo intenso a engordar. Se produce además una alteración de la percepción del peso o la silueta corporal, exagerándose su importancia en la autoevaluación o negándose el peligro que implica la baja de peso corporal.

Las características diagnósticas básicas de la BN consisten en atracones y métodos compensatorios inadecuados para evitar el aumento de peso y en una autoevaluación excesivamente influida por el peso y la silueta corporal.

Respecto a la epidemiología de los TCA, estudios de prevalencia indican que entre el $1 \%$ y el $2 \%$ de mujeres adolescentes padecen AN, aumentando el porcentaje hasta entre un 2 a un $4 \%$ en la BN. Se ha reportado que una de cada dos adolescentes de entre 15 y 16 años "piensa" que su peso es elevado (3).

Aun cuando la prevalencia de los TCA es mayor en las mujeres, la relación de 1 hombre por cada 10 mujeres observada hace años en muestras tanto comunitarias como clínicas ha ido cambiando. Investigaciones recientes han informado relaciones que oscilan entre 5:1 a 2:1, reportándose incluso prevalencias en hombres de un $8 \%$ para la $\mathrm{AN}$ y de alrededor del $15 \%$ para la BN (4).

Los TCA se presentan cada vez a edades más tempranas. En la última década, la edad de aparición ha disminuido en 12 meses, manteniéndose a la vez su prevalencia en edades más avanzas, lo que ha implicado que la progresión de los TCA sea etiquetada como epidémica en sociedades avanzadas (5).

En Chile no existen estudios acabados respecto a la 
prevalencia de estos trastornos. En las bases de datos de revistas indizadas es posible encontrar sólo estudios de riesgo de TCA en: (a) adolescentes escolarizados de la región metropolitana (6), con un riesgo de prevalencia de TCA de $8,3 \%$, (b) en escolares de III y IV medio de la $\mathrm{V}$ región (7), con un riesgo de $18 \%$, (c) en estudiantes de nutrición y dietética (8), una prevalencia de riesgo de $15 \%$ y (d) en tres colegios particulares de Viña del Mar (9), con un riesgo total del $12 \%$. La disparidad de prevalencias de riesgo de un TCA puede deberse fundamentalmente a la diversidad de instrumentos utilizados, de los cuales no se ha reportado las propiedades psicométricas en población chilena, así como la falta de un criterio único para establecer un punto de corte para establecer la categoría de "en riesgo".

El presente estudio tiene como objetivo describir las prevalencias de riesgo de TCA, utilizando un instrumento adaptado para adolescentes chilenos, con un número tal de participantes que disminuye la probabilidad de error y que permite reportar prevalencias de riesgo tanto para hombres como mujeres, además de grupos de edad y tipos de establecimientos educativos, a fin de poder facilitar la focalización de futuras intervenciones en el campo preventivo e intervencional.

Se espera encontrar diferencias significativas en las prevalencias de riesgo dadas por el sexo, en donde las mujeres presenten una prevalencia mayor que los hombres, en la edad, en donde haya una mayor prevalencia a medida que aumente la edad, y al tipo de establecimiento, donde se espera encontrar mayores prevalencias en establecimientos escolares públicos.

\section{SUJETOS Y MÉTODO Tamaño de la muestra}

Considerando una población entre 15 a 29 años cercana a las 79000 (10) personas se calculó un tamaño muestral mínimo de 987 participantes, considerando una prevalencia de riesgo estimada de un $12 \%$, una prevalencia menor aceptable de un $8 \%$ y un nivel de confianza de un $99.99 \%$.

Dado que interesa conocer la prevalencia de riesgo a nivel general, se consideró una muestra no clínica de tipo intencional estratificada. Dada la posibilidad de encontrar datos perdidos y de considerar un número suficiente de cada uno de los estratos, se encuestó a 1429 participantes. La estratificación se realizó intentando cuidar un porcentaje similar de grupos de sexo, pertenencia a tipo de establecimiento educacional y rango de edad (Grupo 1: 13 a 14 años; Grupo 2: 15 a 16 años y Grupo 3: 17 a 18 años). De los participantes 595 fueron hombres $(42 \%)$ y 835 mujeres $(58 \%)(n=1430)$. Durante el año 2008 cursaban entre primero y cuarto medio de enseñanza secundaria en establecimientos educacionales públicos, subvencionados y privados de la ciudad de Antofagasta.

\section{Instrumento}

El Eating Disorder Inventory (EDI) fue creado por Garner (11). Es una escala de auto-reporte que mide características psicológicas y sintomatología asociada a AN y BN. Inicialmente contaba con 64 ítems en 8 subescalas a las cuales fueron adicionados 27 ítems en 3 subescalas, denominándose EDI-2 (12).

La versión en castellano del EDI - 2 consiste en 91 reactivos con un formato de respuestas tipo Likert que permiten puntuar en 11 escalas (13): 8 principales (obsesión por las delgadez, bulimia, insatisfacción corporal, ineficacia, perfeccionismo, desconfianza interpersonal, conciencia introceptiva y miedo a la madurez) y 3 adicionales (acetismo, impulsividad e inseguridad social).

Sobre este instrumento se han reportado características psicométricas adecuadas, tanto en sus versiones en inglés (11), en castellano (14) y en su adaptación a población chilena (15). La utilización de este instrumento ha demostrado ser un buen indicador para discriminar entre pacientes con trastorno alimentario y un grupo control (16).

\section{Procedimientos}

La investigación fue aprobada por el Comité de Ética de la Universidad Católica del Norte y por la Corporación Municipal de Desarrollo Social, organismo que administra los establecimientos educacionales. Una vez solicitados los permisos y autorizaciones necesarias, se aplicó el cuestionario de manera colectiva en cada sala de clases durante la jornada escolar, solicitándose previamente el asentimiento a todos los adolescentes que participarían en el estudio. El tiempo de respuesta osciló de 25 a 45 minutos.

Para su análisis los cuestionarios se ingresaron en una base de datos en SPSS 17.0. Se realizaron análisis de tipo descriptivo por sexo, rango de edad y tipo de establecimiento. Posteriormente se analizaron las diferencias de medias en las conductas evaluadas por el EDI2 entre los grupos, utilizando la prueba T para grupos independientes para sexo y edad y un ANOVA para establecimientos educacionales.

A fin de poder comparar datos con estudios similares en otras regiones del país, se optó por establecer un punto de corte similar al utilizado por Correa et al. en la Región Metropolitana (6), considerándose en la categoría de riesgo los sujetos con un puntaje igual o superior a 110 puntos en la escala total. Este puntaje equivale al percentil 93 en esta muestra y al percentil 
85 en el estudio de Correa, por lo que se asumió habría una reducción del número de falsos positivos con el consecuente aumento de falsos negativos.

\section{RESULTADOS \\ Participantes}

Participaron 1.429 sujetos escolarizados de 13 a 18 años de edad con una media de 15,55 años (D.E.= 1,21). La distribución de los sujetos por curso fue $38 \%$ de primero medio, $22 \%$ segundo medio, $27,6 \%$ de tercero medio y un $12,3 \%$ en cuarto medio.
El $42 \%$ asistía a colegios públicos, $73 \%$ a establecimientos subvencionados y $21 \%$ a establecimientos privados. La distribución por sexo, tipo de establecimiento y rango de edad se presenta en la tabla 1.

\section{Distribución de conductas de riesgo en muestra total y por sexo}

La tabla 2, muestra que las medias de las conductas de riesgo de las mujeres son mayores en casi todas las dimensiones en comparación a los varones. Estas diferencias son estadísticamente significativas en las

\section{TABLA 1}

Distribución de la muestra por sexo y rango de edad según tipo de establecimiento

\begin{tabular}{|c|c|c|c|c|c|c|c|}
\hline & \multicolumn{2}{|c|}{13 a 14 años } & \multicolumn{2}{|c|}{15 a 16 años } & \multicolumn{2}{|c|}{17 a 18 años } & \multirow[t]{2}{*}{ Total } \\
\hline & $\mathbf{H}$ & $\mathbf{M}$ & $\mathbf{H}$ & $\mathbf{M}$ & $\mathbf{H}$ & $\mathbf{M}$ & \\
\hline Público & 58 & 123 & 103 & 162 & 66 & 88 & 600 \\
\hline Subvencionado & 40 & 72 & 118 & 163 & 66 & 72 & 531 \\
\hline Privado & 26 & 41 & 82 & 84 & 36 & 29 & 298 \\
\hline Total & 124 & 236 & 303 & 409 & 168 & 189 & \\
\hline Total grupo edad & 360 & & 712 & & 357 & & 1429 \\
\hline
\end{tabular}

\section{TABLA 2}

Distribución de indicador de riesgo en la muestra total, según sexo

\begin{tabular}{|c|c|c|c|c|c|c|}
\hline & $\begin{array}{l}\text { Muestra total } \\
(1407 a) \\
\text { media }\end{array}$ & d.e & $\begin{array}{l}\text { Hombres } \\
(586) \\
\text { media }\end{array}$ & d.e & $\begin{array}{l}\text { Mujeres } \\
(821) \\
\text { media }\end{array}$ & d.e \\
\hline OD & 6,05 & 5,66 & 3,95 & 4,35 & $7,53^{*}$ & 6,00 \\
\hline B & 2,16 & 3,08 & 2,11 & 2,84 & 2,18 & 3,25 \\
\hline IC & 7,96 & 5,78 & 6,72 & 5,15 & $8,84 *$ & 6,03 \\
\hline MM & 10,54 & 4,43 & 10,47 & 4,61 & 10,59 & 4,31 \\
\hline CI & 5,88 & 5,09 & 5,17 & 4,59 & $6,39 *$ & 5,37 \\
\hline I & 4,74 & 4,73 & 4,20 & 4,41 & $5,13 *$ & 4,91 \\
\hline $\mathrm{P}$ & 6,44 & 3,88 & 6,61 & 3,89 & 6,31 & 3,87 \\
\hline DI & 5,26 & 3,84 & 5,31 & 3,81 & 5,22 & 3,85 \\
\hline IM & 6,76 & 5,60 & 6,68 & 5,31 & 6,83 & 5,80 \\
\hline $\mathrm{A}$ & 6,01 & 2,91 & 5,98 & 2,94 & 6,03 & 2,88 \\
\hline IS & 6,27 & 4,44 & 6,30 & 4,23 & 6,25 & 4,58 \\
\hline
\end{tabular}


dimensiones obsesión por la delgadez $(\mathrm{t}(399)=-12,286$; $\mathrm{p}=.00)$, insatisfacción corporal $(\mathrm{t}(1364)=-6,818 ; \mathrm{p}=.00)$, conciencia introceptiva $(\mathrm{t}(1380)=-4,430 ; \mathrm{p}=.00)$ e ineficacia $(t(1363)=-3,604 ; p=.00)$. En todas ellas, la media fue mayor en las mujeres.

\section{Distribución de conductas de riesgo por edad}

La tabla 3 contiene las medias de las conductas observadas en cada dimensión al estratificar por rango de edad. Se encuentran diferencias significativas en las dimensiones obsesión por la delgadez $(\mathrm{F}(2,1398)=$ 3.787; $\mathrm{P}=, 023)$, bulimia $(\mathrm{F}(2,191)=4.592 ; \mathrm{P}=, 010)$, miedo a la madurez $(\mathrm{F}(2,1379)=16.722 ; \mathrm{P}=, 000)$ y en ineficacia $(\mathrm{F}(2,1362)=4,443 ; \mathrm{P}=, 012)$.

Al aplicar las pruebas Post Hoc usando el estadístico de Bonferroni, arrojó que las diferencias en la dimensión obsesión por la delgadez se encuentran entre los grupos de edad 1 y 3 ( $p=.02$ ), en bulimia la diferencia estuvo entre los grupos 1 y $2(\mathrm{p}=.01)$, en miedo a la madurez las diferencias se observan entre todos los rangos de edad $(\mathrm{p}=.00)$ y finalmente en la dimensión ineficacia existe diferencia entre los grupos 1 y $3(\mathrm{p}=$ $.02)$ y 2 y $3(\mathrm{p}=.03)$.

\section{Distribución de conductas de riesgo por tipo de establecimiento educacional}

Las medias por tipo de establecimiento para cada una de las dimensiones se obervan en la tabla 4. Se encuentran diferencias significativas entre los tipos de establecimientos en las dimensiones obsesión por la delga$\operatorname{dez}(\mathrm{F}(2,1398)=4,607 ; \mathrm{p}=, 010)$, conciencia introceptiva $(\mathrm{F}(2,1379)=14,581 ; \mathrm{p}=, 000)$, ineficacia $(\mathrm{F}(2,1362)=$ 7.616; $\mathrm{p}=, 001)$, perfeccionismo $(\mathrm{F}(2,1364)=4,223$; $\mathrm{p}=$ $, 015)$, desconfiaza interpersonal $(\mathrm{F}(2,1389)=13.354 ; \mathrm{p}=$ ,000), impulsividad $(\mathrm{F}(2,1358)=11,252 ; \mathrm{p}=, 000)$ y en inseguridad social $(\mathrm{F}(2,1373)=8,465 ; \mathrm{p}=, 000)$.

Los análisis Post Hoc utilizando el estadístico de Bonferroni por tipo de establecimiento, se encontraron diferencias de medias en las dimensiones: obsesión por la delgadez $(\mathrm{p}=, 009)$ entre establecimientos públicos y subvencionado; conciencia Introceptiva $(p=, 000)$ entre los tres tipos de establecimientos; ineficacia $(\mathrm{p}=$ ,000) con diferencia entre los públicos y los privados; perfeccionismo $(\mathrm{p}=, 012)$ entre colegios privados $\mathrm{y}$ subvencionados; desconfianza Interpersonal $(p=, 000)$ entre los tres tipos de establecimientos, y en las tres dimensiones complementarias de la escala, existe di-

\section{TABLA 3}

\section{Distribución de conductas de riesgo por edad}

\begin{tabular}{|c|c|c|c|c|c|c|}
\hline & \multicolumn{2}{|c|}{$\begin{array}{c}\text { Grupo } 1 \\
13 \text { a } 14 \text { años } \\
(n=360)\end{array}$} & \multicolumn{2}{|c|}{$\begin{array}{c}\text { Grupo } 2 \\
15 \text { a } 16 \text { años } \\
(n=712)\end{array}$} & \multicolumn{2}{|c|}{$\begin{array}{c}\text { Grupo } 3 \\
17 \text { a } 18 \text { años } \\
(n=357)\end{array}$} \\
\hline & Media & d.e. & Media & d.e. & Media & d.e. \\
\hline OD & $6,55 \mathrm{a}$ & 5,57 & 6,12 & 5,81 & 5,40 & 5,39 \\
\hline B & 1,73 & 2,54 & $2,33 b$ & 3,20 & 2,23 & 3,30 \\
\hline IC & 8,37 & 5,94 & 7,93 & 5,67 & 7,60 & 5,83 \\
\hline MM & $11,60 \mathrm{c}$ & 4,43 & $10,42 \mathrm{a}$ & 4,27 & 9,70 & 4,54 \\
\hline CI & 5,77 & 4,85 & 6,06 & 5,28 & 5,63 & 4,94 \\
\hline I & $5,07 \mathrm{a}$ & 4,38 & 4,90 & 5,03 & 4,10 & 4,38 \\
\hline $\mathrm{P}$ & 6,51 & 3,47 & 6,47 & 3,98 & 6,30 & 4,07 \\
\hline DI & 5,56 & 4,00 & 5,29 & 3,89 & 4,90 & 3,52 \\
\hline IM & 7,26 & 5,55 & 6,67 & 5,65 & 6,44 & 5,53 \\
\hline A & 6,04 & 3,01 & 6,00 & 2,86 & 5,99 & 2,89 \\
\hline IS & 6,26 & 4,49 & 6,45 & 4,50 & 5,92 & 4,24 \\
\hline
\end{tabular}


ferencia en Impulsividad $(\mathrm{p}=, 000)$ entre los tres tipos y finalmente en inseguridad social $(\mathrm{p}=, 000)$ entre colegios privados y públicos.

\section{Frecuencia de Riesgo de TCA}

A nivel de la muestra total, considerando como punto de corte el percentil 93 (110 puntos), se encontró que 1044 participantes no tenían riesgo de desarrollar o tener TCA $(92,6 \%)$, versus 83 personas $(7,4 \%)$ que si lo estaban.

Al estratificar este análisis, se observó que el porcentaje de sujetos en riesgo es mayor en mujeres que en hombres, en los grupos 2 y 3 (15 a 18 años) (tabla 5) y en los participantes de establecimientos educacionales públicos por los subvencionados y privados (tabla 6 ).

La tabla 7 muestra la distribución de los puntajes del grupo riesgo de TCA versus los puntajes del grupo de la categoría de no riesgo en las escalas del EDI2. Tal como se observa, fueron significativamente mayores en todas las dimensiones en los y las adolescentes que fueron categorizados en el grupo de riesgo de TCA.

\section{TABLA 4}

Distribución de conducta de riesgo por tipo de establecimiento

\begin{tabular}{|c|c|c|c|c|c|c|}
\hline \multirow[t]{2}{*}{ Dimensión } & \multicolumn{2}{|c|}{$\begin{array}{l}\text { Públicos (1) } \\
(589)\end{array}$} & \multicolumn{2}{|c|}{$\begin{array}{l}\text { Subvencionados (2) } \\
(517)\end{array}$} & \multicolumn{2}{|c|}{$\begin{array}{l}\text { Privados (3) } \\
(295)\end{array}$} \\
\hline & media & d.e & media & d.e & media & d.e \\
\hline OD & $6,57 b$ & 5,79 & 5,55 & 5,52 & 5,88 & 5,56 \\
\hline B & 2,32 & 3,13 & 1,99 & 3,06 & 2,13 & 3,04 \\
\hline IC & 8,37 & 5,68 & 7,80 & 5,90 & 7,41 & 5,71 \\
\hline MM & 10,78 & 4,34 & 10,47 & 4,59 & 10,18 & 4,33 \\
\hline CI & $6,74 \mathrm{c}$ & 5,19 & $5,21 \mathrm{a}$ & 5,03 & 5,33 & 4,74 \\
\hline I & $5,24 \mathrm{a}$ & 4,88 & 4,62 & 4,80 & 3,94 & 4,14 \\
\hline $\mathrm{P}$ & 6,78 & 3,87 & 6,09 & 3,91 & $6,37 b$ & 3,82 \\
\hline DI & $5,87 \mathrm{c}$ & 3,89 & $4,86 a$ & 3,73 & 4,74 & 3,74 \\
\hline IM & $7,60 \mathrm{c}$ & 5,68 & $6,07 \mathrm{a}$ & 5,50 & 6,30 & 5,40 \\
\hline A & 6,02 & 2,93 & 5,93 & 2,79 & 6,12 & 3,07 \\
\hline IS & $6,74 a$ & 4,59 & 6,21 & 4,44 & 5,45 & 4,00 \\
\hline
\end{tabular}

OD=Obsesión por las delgadez; B=Bulimia; IC=Insatisfacción corporal; I=Ineficacia; P=Perfeccionismo; DI=Desconfianza interpersonal; $\mathrm{CI}=$ Conciencia intropceptiva, $\mathrm{MM}=$ Miedo a la madurez; $\mathrm{A}=$ Acetismo; $\mathrm{IM}=\mathrm{Impulsividad;} \mathrm{IS=Inseguridad} \mathrm{social;}$

$a=$ media significativamente mayor que (3); b= media significativamente mayor (2); $c=$ media significativamente mayor que (2) y (3).

\section{TABLA 5}

Frecuencia y porcentaje de riesgo de trastorno de la conducta alimentaria por sexo y rango de edad

\begin{tabular}{lllllllllll} 
& \multicolumn{2}{c}{ Hombre } & \multicolumn{2}{c}{ Mujer } & \multicolumn{2}{c}{$\mathbf{1 3 - 1 4} \mathbf{a}$} & \multicolumn{2}{c}{$\mathbf{1 5 - 1 6}$ a } & \multicolumn{2}{c}{$\mathbf{1 7 - 1 8 a}$} \\
& F & \% & F & \% & F & \% & F & \% & F & \% \\
\hline SRT & 461 & 94,7 & 583 & 91,1 & 270 & 93,8 & 521 & 92,2 & 253 & 92,3 \\
CRT & 26 & 5,3 & 57 & 8,9 & 18 & 6,3 & 44 & 7,8 & 21 & 7,7 \\
Total & 487 & 100,0 & 640 & 100,0 & 288 & 100,0 & 565 & 100,0 & 274 & 100,0
\end{tabular}

$\mathrm{F}=$ Frecuencia; $\mathrm{SRT}=\mathrm{Sin}$ riesgo trastorno; $\mathrm{CRT}=$ Con riesgo trastorno 


\section{DISCUSIÓN}

La prevalencia de riesgo de trastornos alimentarios encontrada en esta investigación, fue menor a la reportada en investigaciones similares en otras ciudades del país, sin embargo, cabe destacar puede tener una explicación en el punto de corte para establecer la categoría de riesgo.

Tal como se ha reportado en la literatura $(14,17)$, se ha encontrado diferencias significativas entre hombres y mujeres y entre rangos de edad. Las mujeres puntúan más alto en casi todas las dimensiones, otorgando mayor significación a temas estéticos como verse bien, a la búsqueda incesante de la delgadez y con una mayor preocupación al peso además de contestar con mayor frecuencia en aquellos ítems que apuntan al hacer dieta y un mayor miedo a engordar.

Con relación a la edad, los adolescentes entre 13 a 14 años puntúan más en los ítems que componen la dimensión obsesión por la delgadez, miedo a la madurez e ineficacia respecto a los adolescentes mayores, lo cual puede ser explicado por características propias de la etapa evolutiva. Sin embargo, en dimensiones claves, como lo es la de bulimia, es el grupo entre 15 a 16 años el cual puntúa más situando a este grupo con un mayor riesgo. Este hecho es corroborado, al considerar el puntaje total de todas las dimensiones, pues este es el grupo

\section{TABLA 6}

Frecuencia y porcentaje de riesgo de TCA por tipo de establecimiento

\begin{tabular}{lllllll} 
& E.Mun. & \multicolumn{2}{c}{} & E. Subv & \multicolumn{2}{c}{ E.Part. } \\
& F & \% & F & \% & F & $\%$ \\
\hline SRT & 442 & 91,1 & 361 & 94,0 & 241 & 93,4 \\
CRT & 43 & 8,9 & 23 & 6,0 & 17 & 6,6 \\
Total & 485 & 100,0 & 384 & 100,0 & 258 & 100,0 \\
\hline F=Frecuencia; SRT=Sin riesgo trastorno; CRT= Con riesgo trastorno & & & &
\end{tabular}

\section{TABLA 7}

\section{Distribución de grupos con y sin riesgo de tener trastornos de la conducta alimentaria.}

\begin{tabular}{|c|c|c|c|c|c|}
\hline & $\begin{array}{l}\text { Sin rie } \\
(1044) \\
\text { media }\end{array}$ & d.e & $\begin{array}{l}\text { Con ri } \\
(83) \\
\text { media }\end{array}$ & d.e & Prueba T \\
\hline OD & 5,33 & 5,16 & 13,10 & 6,08 & $\mathrm{t}(1125)=-13,001 * *$ \\
\hline B & 1,85 & 2,60 & 5,86 & 5,19 & $\mathrm{t}(1125)=-12,255^{*} *$ \\
\hline IC & 7,08 & 5,05 & 16,10 & 6,18 & $\mathrm{t}(1125)=-15,393 * *$ \\
\hline MM & 10,22 & 4,24 & 13,40 & 5,74 & $\mathrm{t}(1125)=-6,383 * *$ \\
\hline $\mathrm{CI}$ & 4,95 & 4,18 & 14,61 & 5,53 & $\mathrm{t}(1125)=--19,757 * *$ \\
\hline I & 3,84 & 3,67 & 13,02 & 6,83 & $\mathrm{t}(1125)=-20,222 * *$ \\
\hline $\mathrm{P}$ & 6,12 & 3,68 & 10,35 & 4,01 & $\mathrm{t}(1125)=--10,012 * *$ \\
\hline DI & 4,91 & 3,60 & 8,52 & 4,58 & $\mathrm{t}(1125)=--8,596^{* *}$ \\
\hline IM & 5,86 & 4,84 & 15,70 & 6,43 & $\mathrm{t}(1125)=-17,342 * *$ \\
\hline A & 5,72 & 2,63 & 9,51 & 3,48 & $\mathrm{t}(1125)=-12,291 * *$ \\
\hline IS & 5,70 & 3,98 & 11,80 & 5,00 & $\mathrm{t}(1125)=-13,150 * *$ \\
\hline
\end{tabular}


que presenta una mayor cantidad de sujetos en riesgo.

Estos datos son útiles para focalizar intervenciones preventivas o de acción tanto en establecimientos educacionales como desde el sistema de salud que trabajan con estos grupos etáreos. Cabe mencionar que en preguntas complementarias adicionales al test utilizado, se encuentra que en esta muestra un porcentaje importante (entre el 10 y $30 \%$ de los encuestados) había utilizado medios compensatorios como el ejercicio excesivo, la auto-provocación de vómitos, uso de laxantes o diuréticos o ayuno para controlar su peso.

En relación a los tipos de establecimientos educacionales también existieron diferencias significativas similares a las encontradas en la Región Metropolitana (6), en donde existiría una mayor prevalencia de riesgo del trastorno en los colegios públicos municipalizados. En general, los adolescentes encuestados de esta categoría de establecimientos presentarían mayores sentimientos de incapacidad en general, inseguridad, vacío, auto desprecio y falta de control sobre la propia vida con respecto de los adolescentes que proviene de establecimientos particulares.

Una limitación del estudio fue la imposibilidad de realizar un seguimiento de aquellos casos que entraron en la categoría de riesgo, dado el anonimato a la hora de llenar los cuestionarios. Cabe destacar que la sensibilidad del método sólo se puede determinar si se confirman los casos de TCA. En esta investigación, este mismo anonimato no permitió la identificación de casos que pudieran corroborarse a través de la realización de una entrevista clínica o de exámenes físicos complementarios que hubiesen permitido evaluar con certeza la existencia de un diagnóstico clínico de un TCA, por lo que el estudio solo ha podido establecer el riesgo de cursarlo. Dado el carácter de transversal, tampoco fue posible seguir la evolución de los grupos de riesgo como hubiese sido posible bajo un diseño longitudinal.

\section{CONCLUSIÓN}

Se encontraon diferencias significativas en las medias de las dimensiones evaluadas entre hombres y mujeres, por grupos de edad y en los distintos establecimientos educacionales. A nivel general, el 7,4\% de los evaluados se encontró en la categoría de riesgo, aumentando a un $8,9 \%$ en las mujeres, teniendo este grupo una mayor prevalencia de riesgo de trastornos alimentarios, así como el grupo de 15 a 18 años y los participantes de establecimientos educacionales municipalizados.

\section{RESUMEN}

Objetivo: Describir la prevalencia de riesgo de trastornos alimentarios en adolescentes escolarizados de
13 a 18 años. Método: Se aplica la Escala EDI-2 a una muestra intencionada estratificada de 1.429 participantes de la ciudad de Antofagasta pertenecientes a establecimientos educacionales municipalizados, subvencionados y particulares. Resultados: se encuentran diferencias significativas en las medias de las dimensiones evaluadas entre hombres y mujeres, entre grupos de edad y entre los distintos establecimientos educacionales. A nivel general, el 7,4\% de los evaluados se encuentra en la categoría de riesgo, aumentando a un $8,9 \%$ en las mujeres. Conclusiones: la prevalencia de riesgo de trastornos alimentarios es mayor en las mujeres, en el grupo de 15 a 18 años y en los participantes de establecimientos educacionales municipalizados.

Palabras clave: trastorno de la conducta alimentaria, prevalencia de riesgo, anorexia, bulimia.

Dirigir la correspondencia a:

Dr. Alfonso Urzúa M.

Escuela de Psicología

Universidad Católica del Norte

Avenida Angamos 0610. Antofagasta.

Antofagasta, Chile

Email:alurzua@ucn.cl

\section{BIBLIOGRAFÍA}

1. González A. Eating disorders in adolescents. Australian Family Physician 2007; 36: 614-19.

2. American Psychiatric Association. DSM-IV: Diagnostic and statistical Manual of Mental Disorders. Editorial Masson. Washington, DC. 1994.

3. Lambruschini N. Leis R. Trastornos de la conducta alimentaria, Protocolos Nutrición 2002; 361-74. Disponible en http://www.aeped.es/sites/default/ files/documentos/8-TCA.pdf.

4. Gempeler J. Trastornos de la alimentación en hombres: cuatro subtipos clínicos. Rev Colombiana Psiquiatr 2006; 35: 352-61

5. Peláez M. Labrador F. Raich R. Epidemiología de los trastornos de la conducta alimentaria en España: revisión y estado de la cuestión. C Med Psicosom 2004; 71/72: $33-40$

6. Correa V. Zubarew G. Silva P. Romero M. Prevalencia de riesgo de trastornos alimentarios en adolescentes mujeres escolares de la Región Metropolitana. Rev Chil Pediatr 2006; 77: 153-60.

7. Behar R. Trastornos del hábito de comer en adolescentes: aspectos clínicos y epidemiológicos. Rev Méd Chil 1998; 126: 1085 - 92.

8. Behar R. Alviña M. Medinellis A. Tapia P. Trastornos de la conducta alimentaria en estudiantes de la carrera de nutrición y dietética. Rev Chil Nutr 2007; 
34: $298-306$.

9. Behar R. Alviña M. González T. Rivera N. Detección de Actitudes y/o conductas predisponentes a trastornos alimentarios en estudiantes de enseñanza media de tres colegios particulares de viña del mar. Rev Chil Nutr 2007; 34: 240 - 9.

10. Instituto Nacional de Estadísticas de_Chile_Información disponible en página weh http://www.ine. cl/cd2002/cuadros/6/C6_02101.pdf), recuperada el 10 marzo 2009

11. Garner D. Olmstead M. Polivy J. Development and validation of a multidimensional eating disorder inventory for anorexia nervosa and bulimia. Int $\mathbf{J}$ Eat Disord 1983; 2: 15 - 34 .

12. Theil A. Thomas P. Test-retest reliability of the eating disorder inventory 2. J Psychosom Res 2006; 61: $567-9$.
13. García E. Vázquez V. Validez interna y utilidad diagnóstica del Eating Disorder Inventory, en mujeres mexicanas. Salud Pública Mex 2003; 45: 206-10.

14. Corral S. González M. Perena J. Seisdedos N. Manual inventario de trastornos de la conducta alimentaria EDI-2. Madrid: TEA Ediciones. 2006

15. Urzúa A. Castro S. Lillo A. Leal C. Evaluación de los trastornos alimentarios: propiedades psicométricas del test EDI-2 en adolescentes escolarizados(as) de 13 a 18 años. Rev Chil Nutr 2009; 36: 1063 - 73.

16. Nevoren L. Broberg A. Validating the Eating Disorder Inventory-2 (EDI-2) in Sweden. Eat Weight Disord 2001; 6:59-67.

17. Herrero M. Conrado M. Conductas y actitudes hacia la alimentación en una muestra representativa en estudiantes de secundaria. Internat J Clin Health Psychol 2005; 5: 67-83. 